occurs there (Masiello and Biggiogera, 2017) but also demethylation of $5 \mathrm{mC}$ leading to $5 \mathrm{hmC}$ formation. We have detected by EM immunocytochemistry the presence of DNMT3 as well as TET enzymes within this area. Our data strengthens the hypothesis that PR represents one of the most active nuclear areas, where the active focus is mobile. Since only particular conditions allow its study, it will be challenging to work on $\mathrm{PR}$ in vivo via super resolution.

References: Bernhard W (1969) A new staining procedure for electron microscopical cytology. J Ultrastruct Res 27(3):250-265 Masiello I, Biggiogera M (2017) Ultrastructural localization of 5-methylcytosine on DNA and RNA. Cell Mol Life Sci 74:3057-3064 Masiello I, Siciliani S, Biggiogera MHistochem Cell Biol (2018) 150:227-233

doi: http://dx.doi.org/10.7124/bc.000A06

\section{B-6. Testing the biological significance of the nuclear localization of actin}

Péter Borkúti ${ }^{1}$, Izabella Bajusz ${ }^{1}$, Csaba Bajusz $^{1}$, Ildikó Kristó ${ }^{1}$, Zoltán Kovács ${ }^{1}$, Péter Vilmos ${ }^{1}$

\footnotetext{
${ }^{1}$ Biological Research Centre of the Hungarian Academy of Sciences, Szeged, Hungary borkutip@brc.hu
}

In recent years it has become clear that the main cytoskeletal component of eukaryotic cells, Actin is present also in the nucleus. However, there was no attempt so far to investigate the biological significance of this localization therefore, we don't know today how essential is nuclear Actin for the organism. The aim of our work is to understand the importance of nuclear Actin in Drosophila melanogaster. Methods: Mutagenesis via RS-elements, molecular cloning, transgenic animal generation, fly genetics, immunohistochemistry. Results: Out of the six Actin coding genes of Drosophila melanogaster, two (Act5C and Act42A) encode ubiquitously expressed, essential proteins which are also present in the nucleus. We chose Act5C in our experiments because its lack is lethal, and the gene has a simple structure, small size and well characterized regulation. To investigate the biological significance of Actin's nuclear function at the organism level, we created a two-component genetic system of Act5C. One element of this system is an Act5C null-mutant line which lacks the whole protein coding region, and the other element is represented by animals carrying various $\mathrm{Act} 5 \mathrm{C}$ transgenes. The transgenes express different forms of the Act5C protein including one which is tagged with a Nuclear Export Signal (NES). The NES tag dramatically decreases the amount of nuclear Act5C, without damaging the protein's cytoplasmic functions. Our subsequent experiments revealed that the NES-Act5C transgene is able to rescue the lethal phenotype of the nullmutants suggesting that the nuclear localization of Actin is not essential. However, the efficiency of the rescue was apparently lower than in the control experiments. Conclusion: Based on this finding and other results, we hypothesize that Actin has essential role in the nucleus but its nuclear functions are most likely redundantly secured. The ways through which the nuclear functions of Actin are assured are under investigation and will be discussed. 